\title{
Feasibility of percutaneous interbody fusion as a treatment for adjacent vertebral stress fracture of ankylosing spondylitis with intervertebral pseudarthrosis formation
}

This article was published in the following Dove Press journal:

Journal of Pain Research

\author{
Qing-Hua Tian \\ Tao Wang \\ Chun-Gen Wu \\ Ming-Hua Li \\ Ying-Sheng Cheng
}

Department of Diagnostic and Interventional Radiology, Shanghai Jiao Tong University Affiliated Sixth People's Hospital, Shanghai 200233, People's Republic of China
Correspondence: Chun-Gen Wu; Ying-Sheng Cheng Department of Diagnostic and Interventional Radiology, Shanghai Jiao Tong University Affiliated Sixth People's Hospital, No 600 Yishan Road, Shanghai 200233, People's Republic of China

$\mathrm{Tel}+86 \quad 8930177559$

Fax +862138297739

Email649514608@qq.com; chengyingsheng@hotmail.com
Objective: The objective of this study was to evaluate the feasibility of percutaneous interbody fusion (PIF) using bone cement for adjacent vertebral stress fracture of ankylosing spondylitis (AS) with intervertebral pseudarthrosis formation.

Patients and methods: From January 2010 to February 2018, eleven consecutive patients (seven men and four women; median age, 56.09 13.64 years; age range, 33-80 years) who underwent PIF as a treatment for adjacent stress fracture of AS with intervertebral pseudarthrosis formation were retrospectively analyzed. The Visual Analog Scale (VAS) and Oswestry Disability Index (ODI) score were assessed before and after the procedure; meanwhile, the procedure duration, length of hospital stay and complications were assessed. Moreover, anterior/ lateral and computed tomography (CT) scans were utilized for the assessment of bone cement distribution and interbody fusion.

Results: Technical success was achieved in all patients, and they experienced good interbody fusion with bone cement after PIF. Mean VAS scores declined significantly from $8.82 \pm 0.87$ before the procedure to $3.36 \pm 0.671$ day after the procedure and $2.73 \pm 0.651$ month after the procedure, while the mean ODI scores decreased from $82.91 \pm 3.02$ before treatment to $31.64 \pm$ 2.661 day after treatment and 30.00 \pm 3.101 month after treatment. The mean procedure duration was $49.73 \pm 6.12$ minutes (range, $42-65$ minutes). The average length of hospital stay was $7.09 \pm 1.45$ days (range, 5-10 days). Extraosseous cement leakage occurred in one case without causing any clinical complications.

Conclusion: PIF is a feasible therapeutic technique for adjacent vertebral stress fracture of AS with intervertebral pseudarthrosis formation, which can significantly relieve pain and stabilize the fractured spine.

Keywords: percutaneous interbody fusion, adjacent vertebral stress fracture, ankylosing spondylitis, pseudarthrosis, feasibility, visual analog scale, Oswestry Disability Index

\section{Introduction}

Ankylosing spondylitis (AS) is a progressive systemic inflammatory disease that primarily affects the sacroiliac joints and spine. ${ }^{1}$ Spine involvement in AS is characterized by the loss of spinal mobility and vertebral osteoporosis., ${ }^{2,3}$ Because of the brittle nature of the spine in AS, the development of a stress fracture can occur with minor trauma or even unknown trauma. ${ }^{4,5}$ Treatment of adjacent vertebral stress fracture due to AS is complex and challenging, requiring systemic and local therapies that include conservative therapy, medication, percutaneous therapeutic techniques and surgical 
options. The clinical management of symptomatic adjacent vertebral stress fracture of AS with intervertebral pseudarthrosis formation is uniquely challenging because it requires alleviating pain caused by both the fractured vertebral and unstable pseudarthrosis.

Percutaneous vertebroplasty (PVP), as a minimally invasive, safe and effective technique, has gained wide clinical acceptance for the treatment of both primary and glucocorticosteroid-induced osteoporotic vertebral compression fractures..$^{6-9}$ However, PVP for adjacent vertebral stress fracture with intervertebral pseudarthrosis formation due to AS is less known. Recently, we have designed an innovative approach - percutaneous interbody fusion (PIF) - developed from PVP, which has not been reported in the literature thus far to our knowledge, especially for patients with adjacent vertebral stress fracture of AS with intervertebral pseudarthrosis formation. In the present study, we aimed to evaluate the feasibility of PIF by assessing the results of interventional treatment for adjacent vertebral stress fracture due to AS with PIF.

\section{Patients and methods Study design}

This retrospective study was approved by the institutional review board of Shanghai Sixth People's Hospital, and written informed consent was obtained from all individual participants included in the study. All procedures performed were in accordance with the ethical standards of the institutional and/or national research committee and with the 1964 Declaration of Helsinki and its later amendments or comparable ethical standards. From January 2010 to February 2018, patients with adjacent vertebral stress fracture of AS with intervertebral pseudarthrosis formation were recruited from our department to PIF treatment. All patients who were referred for the treatment were asked by a practitioner to complete a short questionnaire about the presence, severity and duration of pain. Patients were eligible for enrollment if they met the following criteria: 1) severe focal pain on the median or paravertebral line, accentuated by digital pressure on the vertebral spinous process at the same level; 2) no radiological evidence of other spinal disorders that could account for the symptoms at the same level; 3 ) reluctant to analgesics and refusal to surgery; 4) absence of neurological signs; 5) with AS and 6) had one clinical and plain film, computed tomography (CT) or magnetic resonance imaging (MRI) follow-up at least 1 month after the initial treatment and diagnosed as having stress fracture with intervertebral pseudarthrosis formation. Patients were excluded if any of the following was present: 1) untreatable coagulopathy; 2) allergy to polymethyl methacrylate (PMMA); 3) severe cardiopulmonary comorbidity; 4) suspected underlying malignant disease or 5) spinal cord compression syndrome.

\section{PIF procedures}

All the procedures were performed by two experienced interventional radiologists (C-GW and TW, with 15 and 10 years of experience in spinal intervention, respectively) on a biplanar unit (Axiom Artis VB22N; Siemens, Berlin, Germany). The patient was placed in a prone position on an operating table. After local anesthesia, 13-gage bone puncture needles (Cook Medical, Bloomington, IN, USA) were used to puncture the adjacent collapsed vertebral bodies through the transpedicular approach, and another 13-gage bone puncture needle (Cook Medical) was slowly inserted at the intended site of entry until the tip reached inside the intervertebral disc cavity. In addition, if required, one or two curved needles were used to pierce through the upper and lower endplates to make better distribution of bone cement. After removal of the inner needle, commercially available PMMA (Osteo-Firm; Cook Medical) was carefully injected into the adjacent vertebral bodies and disk cavity under continuous fluoroscopic monitoring. Injection was stopped if it became difficult because of high resistance or when the cement reached the posterior vertebral wall or entered an extraosseous space. The amount of bone cement used for PIF and operation time were noted. Immediately after the procedure, standard anterior and lateral radiographs were obtained to assess the cement distribution, cement leakage or other possible local complications (Figure 1).

\section{Clinical outcome evaluation and data collection}

Patients were clinically examined by two of the authors (Y-SC and M-HL) who gathered initial and follow-up data before the procedure and 1 day, 1, 3 and 6 months after the treatment and every subsequent 6 months thereafter. In cases where clinical examination could not be performed, patients or their family were contacted by telephone every 3 months. Imaging follow-up comprised anterior/lateral and bending roentgenograms at 1 month, 3 months, 6 months and 1 year after the procedure. CT and/or MRI were performed in the same manner as before the procedure and every 3 months after the procedure in all patients.

The feasibility of this new procedure, the technical success, PMMA volume injected, pain relief, functional outcomes, the length of hospital stay and complications were 

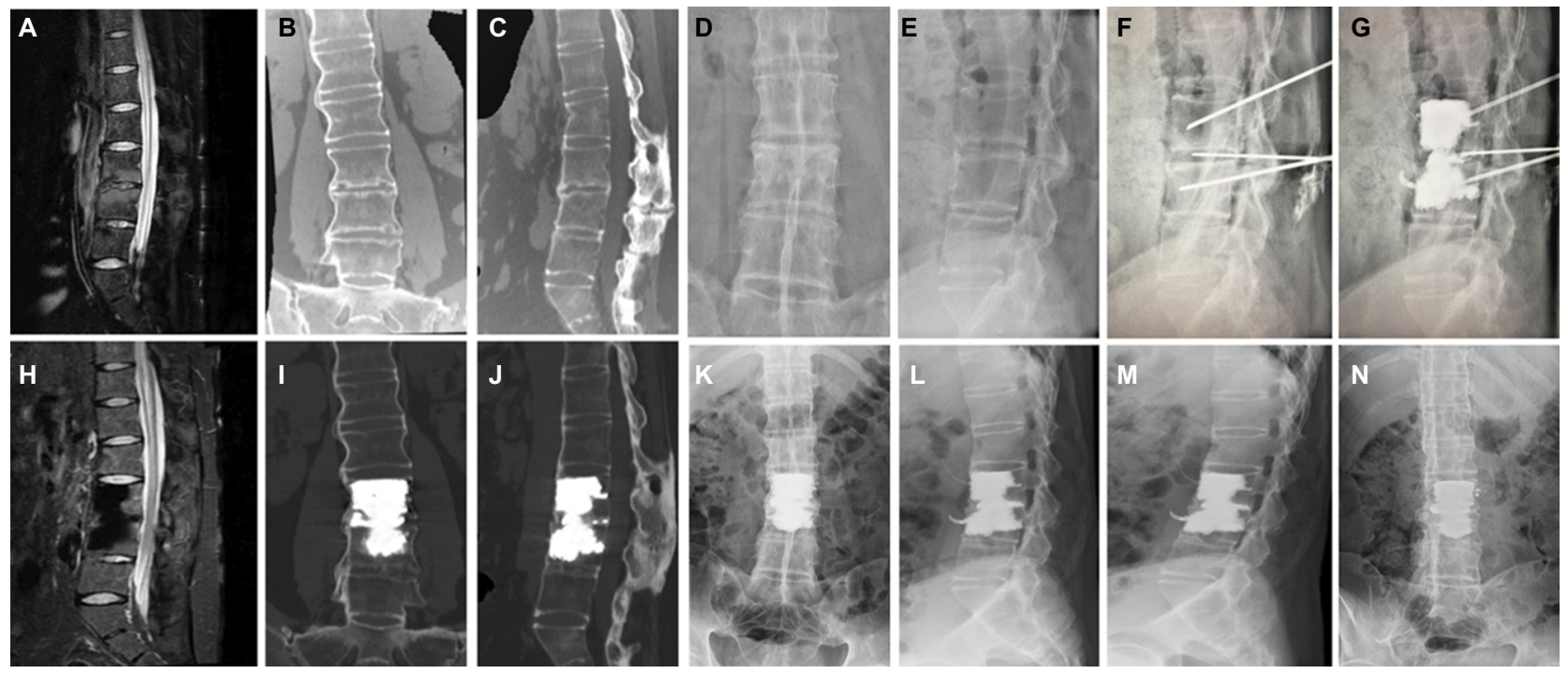

Figure I Adjacent vertebral stress fracture with intervertebral pseudarthrosis formation of the L3-L4 segment owing to AS in a 32-year-old male pre and post PIF. Notes: (A) Preoperative T2WI sagittal MRI images of the patient demonstrating stress fracture with intervertebral pseudarthrosis formation of the L3-L4 segment. (B-E) Preoperative reconstructive $C T$ and anterior/lateral $X$-ray images documenting bone bridging around the disk. (F and $\mathbf{G})$ Intraoperative images showing puncture and bone cement injection for fractured L3-L4 segment. $(\mathbf{H}-\mathbf{N})$ Postoperative T2WI sagittal MRI, reconstructive CT and anterior/lateral and bending roentgenograms of the patient showing good distribution of bone cement and fine fusion of the L3-L4 segment.

Abbreviations: AS, ankylosing spondylitis; PIF, percutaneous interbody fusion; T2WI, T2-weighted image; MRI, magnetic resonance imaging; CT, computed tomography.

evaluated at follow-up consultations or at patient death. Technical success was defined as the successful puncture of the adjacent collapsed vertebral bodies, the successful puncture of the intervertebral disc cavity and the subsequent injection of PMMA without any major complications. The clinical criteria for interbody fusion were assessed by improvement of clinical symptoms, including pain relief and functional status. Moreover, we evaluated the interbody fusion radiologically by means of reconstructive $\mathrm{CT}$ and anterior/lateral and bending roentgenograms. In this study, interbody fusion was defined as making the fractured segments a whole by means of bone puncture and injection of bone cement into the adjacent vertebral bodies and pseudarthrosis. Good interbody fusion was supposed to be bony bridging around the disk and anterior/lateral vertebral bodies and no occurrence of pseudarthrosis. The pain relief was measured by the Visual Analog Scale (VAS) ${ }^{10}$ score ranging from 0 (no pain) to 10 (worst pain ever), while the functional status of patients for walking, standing and sleeping was measured by the Oswestry Disability Index (ODI) ${ }^{11}$ questionnaire. The VAS is a 10-point scale on which patients are asked to rate themselves based on their level of back and/or leg pain, with scores of 0 indicating no pain and 10 indicating the worst pain possible. The ODI is a 10-item ordinal scale for which each item has six possible responses. The score is measured as a percentage $(0 \%-100 \%)$, with an increasing score indicating increasing disability. ODI scores are given as percentages throughout this article. The MacNab criteria were used to access the efficacy of PIF, which define an excellent outcome as no pain and no limitation of normal life; good outcome as occasional pain or paresthesia but no need of medication and no limitation of normal life; fair outcome as somewhat improved pain but a need for medication, with some limitation of normal life; and poor outcome as no improvement or worsening and/or a need for additional surgical treatment due to incomplete decompression.

\section{Statistical analysis}

Descriptive data were given as mean \pm SD. Dichotomous and categorical data were reported as numbers, and descriptive data were represented as mean $\pm \mathrm{SD}$. The paired $t$-test was used to compare the mean VAS and ODI scores between the different study time points; $P \leq 0.05$ was considered as statistically significant. All statistical analyses were performed using SPSS version 16.0 software (SPSS Inc., Chicago, IL, USA).

\section{Results}

A total of eleven consecutive patients (seven men and four women; median age, 56.09 \pm 13.64 years; age range, 33-80 years) were examined in the present study. There was a trauma history in all patients. The demographic and clinical data of patients are listed in Table 1. PIF was technically 


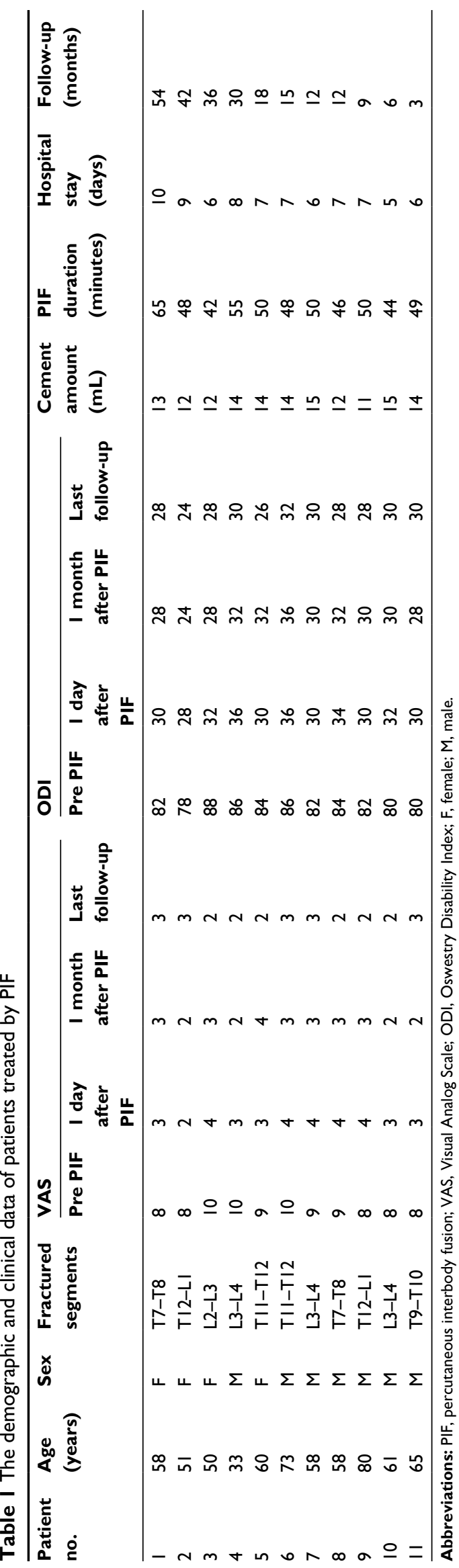

feasible in all patients, and the mean duration of the procedure was $49.73 \pm 6.12$ minutes (range, $42-65$ minutes). The mean volume of PMMA injected was $13.27 \pm 1.35 \mathrm{~mL}$ (range, 11-15 mL), and the average length of hospital stay was $7.09 \pm 1.45$ days (range, $5-10$ days).

The researchers followed up with the patients for 3-54 months, with an average of $21.55 \pm 16.53$ months. All patients experienced good interbody fusion after PIF with bone cement, showing bony bridging around the disk and anterior/ lateral vertebral bodies and no occurrence of pseudarthrosis. According to the MacNab criteria, seven (63.64\%) patients had excellent results, four (36.36\%) had good results and none $(0 \%)$ had fair or poor results after PIF procedure. The mean score of pain on VAS was $8.82 \pm 0.87$ (range, 8-10) before treatment; the pain was relieved immediately after treatment in all eleven patients, with the mean pain score decreasing to $3.36 \pm 0.67$ (range, 2-4) 1 day after treatment, $2.73 \pm 0.65$ (range, 2-4) 1 month after treatment, $2.82 \pm 0.60$ (range, 2-4) 3 months after treatment, 2.70 \pm 0.48 (range, 2-3) 6 months after treatment, $2.63 \pm 0.52$ (range, 2-3) 12 months after treatment, and $2.45 \pm 0.52$ (range, 2-3) at the last follow-up, showing that PIF had a persistent painrelieving effect $(P<0.01$; Tables 1 and 2$)$. The mean ODI score decreased from 82.91 \pm 3.02 (range, 78-88) before the procedure to $31.64 \pm 2.66$ (range, 28-36) 1 day after the procedure, $30.00 \pm 3.10$ (range, 24-36) 1 month after treatment, 29.82 \pm 3.03 (range, 24-36) 3 months after treatment, 29.40 \pm 2.32 (range, 24-32) 6 months after treatment, $28.75 \pm 2.60$ (range, 24-32) 12 months after treatment, and 28.55 \pm 2.21 (range, 24-32) at the last follow-up, remaining low throughout the follow-up period $(P<0.01$; Tables 1 and 2$)$. There were no PIF-related complications after operation, except that one patient had asymptomatic leakage of bone cement into the paravertebral vein.

\section{Discussion}

As the stress fracture level is always the last mobile joint between two ankylosed spinal segments, the continued movement at the fracture site eventually contributes to the development of pseudarthrosis. ${ }^{12}$ The current literature contains proponents of both conservative and surgical management of stress fracture in patients with AS. ${ }^{8}$ Recommendations for conservative treatment generally include prolonged bed rest and early immobilization. Nonetheless, nonoperative measures can result in neurologic deterioration or failed union and is often inadequate, leading many to support surgical fusion as the treatment of choice. A variety of surgical approaches have been used to treat these fractures, 
Table 2 Clinical outcomes of VAS and ODI during follow-ups

\begin{tabular}{llllllll}
\hline Evaluation & Pretreatment & I day & I month & $\mathbf{3}$ months & $\mathbf{6}$ months & I 2 months & Last follow-up \\
\hline VAS & $8.82 \pm 0.87$ & $3.36 \pm 0.67^{*}$ & $2.73 \pm 0.65^{*}$ & $2.82 \pm 0.60^{*}$ & $2.70 \pm 0.48^{*}$ & $2.63 \pm 0.52^{*}$ & $2.45 \pm 0.52^{*}$ \\
ODI & $82.91 \pm 3.02$ & $31.64 \pm 2.66^{*}$ & $30.00 \pm 3.10^{*}$ & $29.82 \pm 3.03^{*}$ & $29.40 \pm 2.32 *$ & $28.75 \pm 2.60^{*}$ & $28.55 \pm 2.2 I^{*}$ \\
\hline
\end{tabular}

Note: $* P<0.01$ compared with that of preoperation at each follow-up point.

Abbreviations: VAS, Visual Analog Scale; ODI, Oswestry Disability Index.

including anterior, posterior and combined approaches with instrumentation three levels above and three levels below the stress fracture..$^{13-15}$

However, the decision to treatment of adjacent vertebral stress fracture with intervertebral pseudarthrosis formation in patients with AS who not only refused surgical treatment but also were resistant to conservative therapy is extremely difficult. Given the fact that reduced mobility and refractory pain are common symptoms in these patients and the pain mainly derived from the instability of pseudarthrosis, we performed PIF for eleven patients in order to relieve pain, stabilize and strengthen spine and improve function. All eleven patients showed immediate mobility regain and pain alleviation after the procedure and did not require any pain medication during follow-up, and there were no clinically significant complications or unwanted side effects. Interestingly, it would take $>1$ hour to wake up for one of the patients before PIF, but 5 minutes was enough after PIF. Another case of patients experienced early and long-lasting pain relief with a follow-up of $>4$ years.

This small series represents a specific group of patients with adjacent vertebral stress fracture of AS with intervertebral pseudarthrosis formation treated with PIF using bone cement. To our knowledge, the role of PIF in patients with adjacent vertebral stress fracture of AS with intervertebral pseudarthrosis formation has not been elucidated in the literature. The authors' early experience with this technique has shown excellent fusion results of adjacent vertebral stress fracture using bone cement. The principle of the method described here is similar to that of PVP. We speculated that the pain relief in our eleven patients with stress fracture due to AS was not only related to the stabilization of fractured segment by bone cement but also to the chemical and thermal effects, which may damage the sensory nerve endings and cytokines as previously reported. ${ }^{16}$ In our opinion, patients with AS were in a state of rigid, painless equilibrium, and this pathological equilibrium was disrupted when stress fracture occurred, followed by pain and disability. However, patients with stress fracture due to AS experienced a new state of painless equilibrium after PIF in spite of sacrificing some minimal movement.

In the present study, PIF was found to be successful and highly feasible, which involves percutaneous puncture of the target spinal segments and injection of bone cement PIF is a minimally invasive procedure, which may be useful for proper selected patients - particularly for those in poor general condition - and has several advantages. First, the procedure is minimally invasive and only requires local anesthesia, which would decrease the risk of infection and blood loss, reduce the risk of general anesthesia and avoid damage to the adjacent tissues, especially the spinal cord. Second, the short period of bed rest required associated with a short hospital stay can certainly decrease the risk of thromboembolic complications.

There are some limitations in the present study. First, the study is limited by its retrospective nature. Second, the number of cases is too small to make broad generalizations. In addition, there is no comparison with other therapeutic options such as surgical treatment. However, to the best of our knowledge, this is the first study to describe PIF for treatment of adjacent vertebral stress fracture of AS with intervertebral pseudarthrosis formation, and our results can serve as a scaffold for the design of future clinical trials.

\section{Conclusion}

Percutaneous stabilization of adjacent vertebral stress fracture with intervertebral pseudarthrosis formation due to AS by using PIF is a highly feasible technique, which can significantly relieve pain and stabilize the fractured spine. Moreover, it appears to be a promising alternative for patients who are not candidates for surgical stabilization and conservative therapy. However, further studies with a larger sample size, which compare this procedure with other available treatments, are required to confirm these preliminary findings.

\section{Acknowledgments}

This work was sponsored by grant National Natural Scientific Fund of China (grant number 81701798), Natural Fund of Shanghai Science and Technology Commission (grant number 18ZR1429400), Shanghai Sailing Program (grant number 17YF1414600), and Shanghai Jiao Tong University Affiliated Sixth People's Hospital East Campus (grant number 2016014), China. 


\section{Disclosure}

The authors report no conflicts of interest in this work.

\section{References}

1. Calin A, Fries JF. Striking prevalence of ankylosing spondylitis in "healthy" W27 positive males and females. NEngl J Med. 1975;293(17): 835-839.

2. Bronson WD, Walker SE, Hillman LS, Keisler D, Hoyt T, Allen SH. Bone mineral density and biochemical markers of bone metabolism in ankylosing spondylitis. J Rheumatol. 1998;25:929-935.

3. El Maghraoui A, Borderie D, Cherruau B, Edouard R, Dougados M, Roux C. Osteoporosis, body composition, and bone turnover in ankylosing spondylitis. J Rheumatol. 1999;26(10):2205-2209.

4. Taggard DA, Traynelis VC. Management of cervical spinal fractures in ankylosing spondylitis with posterior fixation. Spine (Phila Pa 1976). 2000;25(16):2035-2039.

5. Vosse D, Feldtkeller E, Erlendsson J, Geusens P, van der Linden S. Clinical vertebral fractures in patients with ankylosing spondylitis. J Rheumatol. 2004;31(10):1981-1985.

6. Balkarli H, Demirtas H, Kilic M, Ozturk I. Treatment of osteoporotic vertebral compression fractures with percutaneous vertebroplasty under local anesthesia: clinical and radiological results. Int J Clin Exp Med. 2015;8(9):16287-16293.

7. Nieuwenhuijse MJ, van Erkel AR, Dijkstra PD. Percutaneous vertebroplasty in very severe osteoporotic vertebral compression fractures: feasible and beneficial. J Vasc Interv Radiol. 2011;22(7): $1017-1023$.
8. Maggi L, Faragò G, Mantegazza R, Ciceri E. Percutaneous vertebroplasty in a series of myasthenic patients with steroid-induced symptomatic vertebral fractures. Neurol Sci. 2013;34(5):773-776.

9. Tian QH, Wu CG, Xiao QP, et al. Percutaneous vertebroplasty of the entire thoracic and lumbar spines for vertebral compression fractures related to chronic glucocorticosteriod use: case report and review of literature. Korean J Radiol. 2014;15(6):797-801.

10. Fairbank J, Pynsent P. The Oswestry Disability Index. Spine. 2000;25:2940-2953.

11. Zanoli G, Stromqvist B, Jonsson B. Visual analog scales for interpretation of back and leg pain intensity in patients operated for degenerative lumbar spine disorders. Spine. 2001;26(21):2375-2380.

12. Bron JL, de Vries MK, Snieders MN, van der Horst-Bruinsma IE, van Royen BJ. Discovertebral (Andersson) lesions of the spine in ankylosing spondylitis revisited. Clin Rheumatol. 2009;28(8):883-892.

13. Kouyoumdjian P, Guerin P, Schaelderle C, Asencio G, Gille O. Fracture of the lower cervical spine in patients with ankylosing spondylitis: retrospective study of 19 cases. Orthop Traumatol Surg Res. 2012;98(5):543-551.

14. Nayak NR, Pisapia JM, Abdullah KG, Schuster JM. Minimally invasive surgery for traumatic fractures in ankylosing spinal diseases. Global Spine J. 2015;5(4):266-273.

15. Xiang LB, Yu HL, Liu J, Chen Y, Yang HF. One-stage surgery by a combined anterior-posterior approach to treat ankylosing spondylitis complicated by a multiple-level cervical vertebral chance fracture. Mod Rheumatol. 2015;25(2):282-285.

16. Lim TH, Brebach GT, Renner SM, et al. Biomechanical evaluation of an injectable calcium phosphate cement for vertebroplasty. Spine. 2002;27(12):1297-1302.
Journal of Pain Research

\section{Publish your work in this journal}

The Journal of Pain Research is an international, peer reviewed, open access, online journal that welcomes laboratory and clinical findings in the fields of pain research and the prevention and management of pain. Original research, reviews, symposium reports, hypothesis formation and commentaries are all considered for publication.

\section{Dovepress}

The manuscript management system is completely online and includes a very quick and fair peer-review system, which is all easy to use. Visit http://www.dovepress.com/testimonials.php to read real quotes from published authors. 